\title{
CHARACTERIZATION OF ALUTHGE TRANSFORM OF COMPOSITION OPERATORS
}

\author{
MORTEZA SOHRABI
}

\begin{abstract}
Let $\widetilde{C_{\varphi}}$ be the Aluthge transform of composition operator on $L^{2}(\Sigma)$. The main result of this paper is characterizations of Aluthge transform of composition operators in some operator classes that are weaker than hyponormal, such as hyponormal, quasihyponormal, paranormal, *-paranormal on $L^{2}(\Sigma)$. Moreover, to explain the results, we provide several useful related examples to show that $\widetilde{C_{\varphi}}$ lie between these classes.
\end{abstract}

MSC(2010): 47B33; 47B38.

Keywords: Aluthge transform, polar decomposition, conditional expectation, hyponormal, paranormal.

\section{Introduction and Preliminaries}

Let $(X, \Sigma, \mu)$ be a sigma finite measure space and let $\mathcal{A}$ be a subsigma algebra of $\Sigma$. We understand $L^{2}(\mathcal{A})$ as a subspace of $L^{2}(\Sigma)$ and as a Banach space. We use the notation $L^{2}(\mathcal{A})$ for $L^{2}\left(X, \mathcal{A}, \mu_{\left.\right|_{\mathcal{A}}}\right)$. Throughout this paper, we assume that all subsigma algebras under consideration are complete and sigma finite. We denote the linear space of all complex-valued $\Sigma$-measurable functions on $X$ by $L^{0}(\Sigma)$. For $f \in L^{0}(\Sigma)$, we define the support of a measurable function $f$ as $\sigma(f)=\{x \in X: f(x) \neq 0\}$. Let $\varphi$ be a mapping from $X$ into $X$ which is measurable, (i.e., $\varphi^{-1}(\Sigma) \subseteq \Sigma$ ) such that $\mu \circ \varphi^{-1}$ is absolutely continuous with respect to $\mu\left(\mu \circ \varphi^{-1} \ll \mu\right)$. Suppose that $h$ is the Radon-Nikodym derivative $d \mu \circ \varphi^{-1} / d \mu$. The composition operator $C_{\varphi}: L^{2}(\Sigma) \rightarrow L^{0}(\Sigma)$ induced by $\varphi$ is given by $C_{\varphi}(f)=f \circ \varphi$, for each $f \in L^{2}(\Sigma)$. Here, the non-singularity of $\varphi$ guarantees that $C_{\varphi}$ is well defined. It is well known fact that for $u \in L^{0}(\Sigma)$, the multiplication operator $M_{u}: L^{2}(\Sigma) \rightarrow L^{0}(\Sigma)$ is bounded if and only if $u \in L^{\infty}(\Sigma)$, and in this case, $\left\|M_{u}\right\|=\|u\|_{\infty}$. Now, by the change of variables formula; $\int_{X}|f \circ \varphi|^{2} d \mu=\int_{X} h|f|^{2} d \mu,\left\|C_{\varphi} f\right\|_{2}=\left\|M_{\sqrt{h}} f\right\|_{2}$ for each $f \in L^{2}(\Sigma)$. It follows that $C_{\varphi}$ maps $L^{2}(\Sigma)$ boundedly into itself, if and only if $h \in L^{\infty}(\Sigma)$,

Date: Received: January 7, 2020, Accepted: April 24, 2021. 
and in this case, $\left\|C_{\varphi}\right\|=\|h\|_{\infty}^{\frac{1}{2}}$. Some other basic facts about composition operators can be found in [9, 20, 22].

Associated with each sigma algebra $\mathcal{A} \subseteq \Sigma$, there exists an operator $E(. \mid \mathcal{A})=E^{\mathcal{A}}($.$) on the set of all non-negative measurable functions f$ or on the set of all functions $f \in L^{2}(\Sigma)$, that is uniquely determined by the conditions

(i) $E^{\mathcal{A}}(f)$ is $E^{\mathcal{A}}$-measurable,

(ii) If $A$ is any $\mathcal{A}$-measurable set for which $\int_{A} f d \mu$ exists, we have

$$
\int_{A} f d \mu=\int_{A} E(f) d \mu \text {. }
$$

The operator $E^{\mathcal{A}}$ is called the conditional expectation operator. The role of conditional expectation operator is important in this note. We list here some of its useful properties:

$\mathrm{E}(1)$ If $f$ is an $E^{\mathcal{A}}$-measurable function, then $E^{\mathcal{A}}(f g)=f E^{\mathcal{A}}(g)$;

$\mathrm{E}(2)$ If $f \geq 0$ then $E^{\mathcal{A}}(f) \geq 0$; If $f>0$ then $E^{\mathcal{A}}(f)>0$;

$\mathrm{E}(3) E^{\mathcal{A}}(1)=1$

$\mathrm{E}(4) E^{\mathcal{A}}\left(|f|^{2}\right)=\left|E^{\mathcal{A}}(f)\right|^{2}$ if and only if $f \in L(\mathcal{A})$;

$\mathrm{E}(5) \int_{\varphi^{-1} A} g f \circ \varphi d \mu=\int_{A} h E^{\varphi^{-1}(\Sigma)}\left(g \circ \varphi^{-1}\right) f d \mu$, for all $g \in L^{2}(\Sigma), A \in \Sigma$.

As an operator on $L^{2}(\Sigma), E^{\mathcal{A}}$ is the contractive orthogonal projection onto $L^{2}(\mathcal{A})$. Take $\mathcal{A}=\varphi^{-1}(\Sigma)$. So for each function $f$ in $L^{2}(\Sigma)$ there is a $\Sigma$-measurable function $F$ such that $E^{\varphi^{-1}(\Sigma)} f=F \circ \varphi$. Moreover, $F$ is uniquely determined in $\sigma(h)$ (see [3]). Therefore, even though $\varphi$ is not invertible the expression $F=\left(E^{\varphi^{-1}(\Sigma)} f\right) \circ \varphi^{-1}$ is well defined. Note that domain of $E^{\mathcal{A}}$ contains $L^{2}(\Sigma) \cup\left\{f \in{ }^{0}(\Sigma): f \geq 0\right\}$. A result, Lambert and Hoover [11] shows that the adjoint $C_{\varphi}{ }^{*}$ of $C_{\varphi}$ on $L^{2}(\Sigma)$ is given by $C_{\varphi}{ }^{*}(f)=h E^{\varphi^{-1}(\Sigma)}(f) \circ \varphi^{-1}$. From this it easily follows that $C_{\varphi}{ }^{*} C_{\varphi}=M_{h}$ and $C_{\varphi} C_{\varphi}{ }^{*}=M_{h \circ \varphi} E^{\varphi^{-1}(\Sigma)}$. The product $M_{u} \circ \varphi$ of $M_{u}$ and $C_{\varphi}$ is called a weighted composition operator,denoted by $W$, with

$$
\|W f\|_{2}=\left\|\sqrt{h E\left(|u|^{2}\right) \circ \varphi^{-1}} f\right\|_{2} .
$$

Put $J=h E\left(|u|^{2}\right) \circ \varphi^{-1}$. It follows that $W$ is bounded on $L^{2}(\Sigma)$ if and only if $J \in L^{\infty}(\Sigma)$ (see [11] and also [3] for a discussion of $E(\cdot) \circ \varphi^{-1}$ when $\varphi$ is not invertible). We shall frequently use the following general properties of $E^{\mathcal{A}}$ and $C_{\varphi}$ acting on $L^{2}(\mathcal{A})$. For a deeper study of some other basic the properties of $E$ see [10, 16, 18].

Let $\mathcal{H}$ be a separable complex Hilbert space and $B(\mathcal{H})$ denote the algebra of all bounded linear operators acting on $\mathcal{H}$. An operator $T \in B(\mathcal{H})$ has a unique polar decomposition $T=U|T|$, where $|T|=\sqrt{T^{*} T}$ is a positive operator and $U$ is a partial isometry satisfying $U U^{*} U=U$ and $\operatorname{KerU}=\operatorname{Ker} T=\operatorname{Ker}|T|, \operatorname{Ker} U^{*}=\operatorname{Ker} T^{*}$. It is known that the parts 
of the polar decomposition $U,\left|C_{\varphi}\right|$ for $C_{\varphi}$ are given by $U=M_{1 / \sqrt{h \circ \varphi}} C_{\varphi}$, $\left|C_{\varphi}\right|=M_{\sqrt{h}}$.

It is a classical fact that the polar decomposition of $T^{*}$ is $U^{*}\left|T^{*}\right|$, where $\left|C_{\varphi}^{*}\right|=M_{\sqrt{h \circ \varphi}} E$ and $U^{*}=\sqrt{h} E(f) \circ \varphi^{-1}$. In [1], A. Aluthge introduced the operator $\widetilde{T}=|T|^{1 / 2} U|T|^{1 / 2}$ for an operator $T \in B(\mathcal{H})$ with its polar decomposition $T=U|T|$ which is called Aluthge transform of $T$. There are a lot of other known properties of Aluthge transform, for important properties see $[8,14,15,25]$.

Composition operators as an extension of shift operators are a good tool for separating weak hyponormal classes. Classic seminormal (weighted) composition operators have been extensively studied by Harrington and whitley [9, 22], Lambert [11, 16], Singh [20], Campbell [3, 4] and Stochel [6]. In [2] some weak hyponormal classes of composition operators are studied. In those work, examples were given which show that composition operators can be used to separate each partial normality class from quasinormal through $w$-hyponormal. But in some cases composition operators can not be separated some of these classes. Hence, it is better that we consider the weighted case of composition operators. In [12] and [7], the authors generalized the work done in [2] and have obtained some characterizations of related $p$-hyponormal weighted composition operators as separately. In [7] some related examples were presented to show that weighted composition operators separate those classes. This note consists of the following. In Section 2 we characterize some weak hyponormal and weak paranormal classes of Aluthge transform of composition operators on $L^{2}(\Sigma)$. Also, we provide several useful related examples to illustrating these classes.

\section{Main Result}

Recall that an operator $T \in B(\mathcal{H})$ is said to be hyponormal if $\left(T^{*} T\right) \geq$ $\left(T T^{*}\right)$ and $T$ is said to be quasihyponormal if $T^{*}\left(T^{*} T\right) T \geq T^{*}\left(T T^{*}\right) T$. For all $x \in \mathcal{H}$, if $\left\|T^{2} x\right\| \geq\|T x\|^{2}$, then $T$ is called a paranormal operator and $T$ is *-paranormal operator if $\left\|T^{2} x\right\| \geq\left\|T^{*} x\right\|^{2}$.

In the following, we investigate characterizations of Aluthge transform of composition operators in some operator classes such as, hyponormal, quasihyponormal, paranormal, *-paranormal. First we need the next proposition.

Proposition 2.1. [21] Let $C_{\varphi} \in B\left(L^{2}(\Sigma)\right)$. Then the following assertions hold:

(i) $\widetilde{C_{\varphi}} f=\sqrt[4]{\frac{h}{h \circ \varphi}} C_{\varphi} f$. 
(ii) Let $U_{\varphi}\left|\widetilde{C_{\varphi}}\right|$ be the polar decomposition of $\widetilde{C_{\varphi}}$. Then

$$
\begin{aligned}
\left|\widetilde{C_{\varphi}}\right|(f) & =\sqrt{h E\left(\frac{h}{h \circ \varphi}\right)^{\frac{1}{2}} \circ \varphi^{-1}} f ; \\
U_{\varphi}(f) & =\frac{\sqrt[4]{h}}{\sqrt{h \circ \varphi E(\sqrt{h})}} f \circ \varphi .
\end{aligned}
$$

Proposition 2.1 has led almost immediately to the following result.

Proposition 2.2. Let $\widetilde{C_{\varphi}} \in B\left(L^{2}(\Sigma)\right)$. Then the following hold.

(i) ${\widetilde{C_{\varphi}}}^{*} f=\sqrt[4]{h^{3}} E(\sqrt[4]{h} f) \circ \varphi^{-1}$.

(ii) $\widetilde{C_{\varphi}}{\widetilde{C_{\varphi}}}^{*} f=\sqrt[4]{h\left(h^{2} \circ \varphi\right)} E(\sqrt[4]{h} f)$.

Proof. (i) Suppose that $g \in L^{2}(\Sigma)$. By Proposition 2.1(i), we have

$$
\begin{aligned}
\left\langle{\widetilde{C_{\varphi}}}^{*} f, g\right\rangle & =\left\langle f, \widetilde{C_{\varphi}} g\right\rangle=\int_{X} f \widetilde{\widetilde{C}_{\varphi}} g d \mu \\
& =\int_{X} \sqrt[4]{\frac{h}{h \circ \varphi}} f \bar{g} \circ \varphi d \mu \\
& =\int_{X} \frac{E(\sqrt[4]{h} f)}{\sqrt[4]{h \circ \varphi}} \bar{g} \circ \varphi d \mu \\
& =\int_{X} \frac{h E(\sqrt[4]{h} f) \circ \varphi^{-1}}{\sqrt[4]{h}} d \mu \\
& =\left\langle\sqrt[4]{h^{3}} E(\sqrt[4]{h} f) \circ \varphi^{-1}, g\right\rangle .
\end{aligned}
$$

Hence, (i) holds.

(ii) By direct computation, we have

$$
\begin{aligned}
& \widetilde{C_{\varphi}}{\widetilde{C_{\varphi}}}^{*} f=\sqrt[4]{\frac{h}{h \circ \varphi}}\left(\sqrt[4]{h^{3}} E(\sqrt[4]{h} f) \circ \varphi^{-1}\right) \circ \varphi \\
& =\sqrt[4]{h\left(h^{2} \circ \varphi\right)} E(\sqrt[4]{h} f) .
\end{aligned}
$$

Therefore, (ii) holds.

Theorem 2.3. Let $\widetilde{C_{\varphi}} \in B\left(L^{2}(\Sigma)\right)$. Then $\widetilde{C_{\varphi}}$ is hyponormal if and only if

$$
\sqrt[4]{h} E(\sqrt{h}) \circ \varphi^{-1} \geq \sqrt[4]{\left(h^{2} \circ \varphi\right)} E(\sqrt[4]{h}) .
$$

Proof. Suppose that $f \in L^{2}(\Sigma)$. We know that,

$$
\begin{aligned}
& \widetilde{C_{\varphi}}{\widetilde{C_{\varphi}}}^{*} f=\sqrt[4]{h\left(h^{2} \circ \varphi\right)} E(\sqrt[4]{h} f), \\
& {\widetilde{C_{\varphi}}}^{*} \widetilde{C_{\varphi}} f=\sqrt{h} E(\sqrt{h}) \circ \varphi^{-1} f \text {. }
\end{aligned}
$$


Thus, $\widetilde{C_{\varphi}}$ is hyponormal if and only if

$$
\left\langle\left({\widetilde{C_{\varphi}}}^{*} \widetilde{C_{\varphi}}-\widetilde{C_{\varphi}}{\widetilde{C_{\varphi}}}^{*}\right) f, f\right\rangle \geq 0 .
$$

But, because $(X, \mathcal{A}, \mu)$ is a $\sigma$-finite measure space, let $f=\chi_{\varphi^{-1} B}$ with $\mu\left(\varphi^{-1} B\right)<\infty$. Then above inner product is non-negative if and only if

$$
\int_{\varphi^{-1} B}\left\{\sqrt{h} E(\sqrt{h}) \circ \varphi^{-1} f-\sqrt[4]{h\left(h^{2} \circ \varphi\right)} E(\sqrt[4]{h} f)\right\} d \mu \geq 0 .
$$

Equivalently,

$$
\int_{\varphi^{-1} B}\left\{\sqrt{h} E(\sqrt{h}) \circ \varphi^{-1}\left(\chi_{B} \circ \varphi\right)-\sqrt[4]{h\left(h^{2} \circ \varphi\right)} E\left(\sqrt[4]{h} \chi_{B} \circ \varphi\right)\right\} d \mu \geq 0 .
$$

Since $E\left(\chi_{B} \circ \varphi\right) \circ \varphi^{-1}=\chi_{B}$ on $\sigma(h)$, using the change variable theorem we deduce that the above integral is equivalent to

$$
\int_{B}\left\{\sqrt{h \circ \varphi^{-1}} E(\sqrt{h}) \circ \varphi^{-2} \chi_{B}-\sqrt[4]{h^{2}\left(h \circ \varphi^{-1}\right)} E(\sqrt[4]{h}) \circ \varphi^{-1} \chi_{B}\right\} h d \mu \geq 0 .
$$

Equivalently,

$$
\int_{B}\left\{\sqrt{h \circ \varphi^{-1}} E(\sqrt{h}) \circ \varphi^{-2}-\sqrt[4]{h^{2}\left(h \circ \varphi^{-1}\right)} E(\sqrt[4]{h}) \circ \varphi^{-1}\right\} h d \mu \geq 0 .
$$

But this is equivalent to $\sqrt[4]{h} E(\sqrt{h}) \circ \varphi^{-1} \geq \sqrt[4]{\left(h^{2} \circ \varphi\right)} E(\sqrt[4]{h})$.

Hence, the proof is complete.

Theorem 2.4. Let $\widetilde{C_{\varphi}} \in B\left(L^{2}(\Sigma)\right)$. Then $\widetilde{C_{\varphi}}$ is quasihyponormal if and only if

$$
E\left\{\frac{E(\sqrt{h}) \circ \varphi^{-1}}{\sqrt[4]{h}}\right\} \geq \sqrt{h \circ \varphi} .
$$

Proof. Suppose that $f \in L^{2}(\Sigma)$. It is easy verify that,

$$
\begin{aligned}
& {\widetilde{C_{\varphi}}}^{*}\left(\widetilde{C_{\varphi}}{\widetilde{C_{\varphi}}}^{*}\right) \widetilde{C_{\varphi}} f=h E(h) \circ \varphi^{-1} f, \\
& {\widetilde{C_{\varphi}}}^{*}\left({\widetilde{C_{\varphi}}}^{*} \widetilde{C_{\varphi}}\right) \widetilde{C_{\varphi}} f=\sqrt{h} E\left\{\sqrt[4]{h^{3}} E(\sqrt{h}) \circ \varphi^{-1}\right\} \circ \varphi^{-1} f .
\end{aligned}
$$

Thus, ${\widetilde{C_{\varphi}}}^{*}\left(\widetilde{C_{\varphi}}{ }^{*} \widetilde{C_{\varphi}}\right) \widetilde{C_{\varphi}} \geq \widetilde{C_{\varphi}}\left(\widetilde{C_{\varphi}}{\widetilde{C_{\varphi}}}^{*}\right) \widetilde{C_{\varphi}}$ if and only if

$$
\sqrt{h} E\left\{\sqrt[4]{h^{3}} E(\sqrt{h}) \circ \varphi^{-1}\right\} \circ \varphi^{-1} \geq h E(h) \circ \varphi^{-1} .
$$

Equivalently,

$$
E\left\{\frac{E(\sqrt{h}) \circ \varphi^{-1}}{\sqrt[4]{h}}\right\} \geq \sqrt{h \circ \varphi} .
$$


Hence the theorem is proved.

Lemma 2.5. Let $T \in B(\mathcal{H})$ and let $U|T|$ be its polar decomposition. Then the following hold:

(i) [17] $T$ is *-paranormal if and only if for each $k>0$,

$$
|T| U^{*}|T|^{2} U|T|-2 k\left|T^{*}\right|^{2}+k^{2} \geq 0 .
$$

(ii) [24] $T$ is paranormal if and only if for each $k>0$,

$$
|T| U^{*}|T|^{2} U|T|-2 k|T|^{2}+k^{2} \geq 0 .
$$

Theorem 2.6. Let $\widetilde{C_{\varphi}} \in B\left(L^{2}(\Sigma)\right)$. Then the following statements hold.

(i) $\widetilde{C_{\varphi}}$ is *-paranormal if and only if

$$
E\left\{h E(\sqrt{h}) \circ \varphi^{-1}\right\} \geq\left(h \circ \varphi^{2}\right) \sqrt{E(\sqrt{h})}\{E(\sqrt[4]{h}) \circ \varphi\}^{2}
$$

on $\sigma(h)$.

(ii) $\widetilde{C_{\varphi}}$ is paranormal if and only if

$$
E\left\{h E(\sqrt{h}) \circ \varphi^{-1}\right\} \geq \sqrt{h \circ \varphi}\{E(\sqrt{h})\}^{\frac{5}{2}}
$$

on $\sigma(h)$.

Proof. (i) Suppose that $f \in L^{2}(\Sigma)$. It is easy to verify that

$$
\begin{aligned}
\left|\widetilde{C_{\varphi}}\right|^{2} f & =\sqrt{h} E(\sqrt{h}) \circ \varphi^{-1} f \\
U_{\varphi}^{*} f & =\sqrt{h} E\left\{\frac{\sqrt[4]{h} f}{E(\sqrt{h})}\right\} \circ \varphi^{-1} . \\
\left|{\widetilde{C_{\varphi}}}^{*}\right|^{2} f & =\widetilde{C_{\varphi}}{\widetilde{C_{\varphi}}}^{*} f=\sqrt[4]{h\left(h^{2} \circ \varphi\right)} E(\sqrt[4]{h} f) .
\end{aligned}
$$

By Proposition 2.1 and above relations, we have

$$
\left|\widetilde{C_{\varphi}}\right| U_{\varphi}^{*}\left|\widetilde{C_{\varphi}}\right|^{2} U_{\varphi}\left|\widetilde{C_{\varphi}}\right| f=\frac{\sqrt{h} E\left\{h E(\sqrt{h}) \circ \varphi^{-1}\right\} \circ \varphi^{-1}}{\sqrt{E(\sqrt{h}) \circ \varphi^{-1}}} f .
$$

By Lemma $2.5(\mathrm{i}), \widetilde{C_{\varphi}}$ is $*$-paranormal if and only if

$$
\left\langle\frac{\sqrt{h} E\left\{h E(\sqrt{h}) \circ \varphi^{-1}\right\} \circ \varphi^{-1}}{\sqrt{E(\sqrt{h}) \circ \varphi^{-1}}} f-2 k \sqrt[4]{h\left(h^{2} \circ \varphi\right)} E(\sqrt[4]{h} f)+k^{2}, f\right\rangle \geq 0
$$


for each $k \in(0, \infty)$. Put $f=\chi_{\varphi^{-1} B}$ with $\mu\left(\varphi^{-1} B\right)<\infty$. Hence, (2.1) holds if and only if

$$
\int_{\varphi^{-1} B}\left\{\frac{\sqrt{h} E\left\{h E(\sqrt{h}) \circ \varphi^{-1}\right\} \circ \varphi^{-1}}{\sqrt{E(\sqrt{h}) \circ \varphi^{-1}}}\left(\chi_{B} \circ \varphi\right)-2 k \sqrt[4]{h\left(h^{2} \circ \varphi\right)} E\left(\sqrt[4]{h} \chi_{B} \circ \varphi\right)+k^{2}\right\} d \mu \geq 0
$$

if and only if

$$
\int_{B}\left\{\frac{\sqrt{h} \circ \varphi^{-1} E\left\{h E(\sqrt{h}) \circ \varphi^{-1}\right\} \circ \varphi^{-2}}{\sqrt{E(\sqrt{h}) \circ \varphi^{-2}}} \chi_{B}-2 k \sqrt[4]{h^{2}\left(h \circ \varphi^{-1}\right)} E(\sqrt[4]{h}) \circ \varphi^{-1} \chi_{B}+k^{2}\right\} h d \mu \geq 0 .
$$

Equivalently,

$$
\int_{B}\left\{\frac{\sqrt{h} \circ \varphi^{-1} E\left\{h E(\sqrt{h}) \circ \varphi^{-1}\right\} \circ \varphi^{-2}}{\sqrt{E(\sqrt{h}) \circ \varphi^{-2}}}-2 k \sqrt[4]{h^{2}\left(h \circ \varphi^{-1}\right)} E(\sqrt[4]{h}) \circ \varphi^{-1}+k^{2}\right\} h d \mu \geq 0 .
$$

But, This is equivalent to

$$
\frac{\sqrt{h} \circ \varphi^{-1} E\left\{h E(\sqrt{h}) \circ \varphi^{-1}\right\} \circ \varphi^{-2}}{\sqrt{E(\sqrt{h}) \circ \varphi^{-2}}}-2 k \sqrt[4]{h^{2}\left(h \circ \varphi^{-1}\right)} E(\sqrt[4]{h}) \circ \varphi^{-1}+k^{2} \geq 0 .
$$

Put

and

$$
a:=\frac{\sqrt{h} \circ \varphi^{-1} E\left\{h E(\sqrt{h}) \circ \varphi^{-1}\right\} \circ \varphi^{-2}}{\sqrt{E(\sqrt{h}) \circ \varphi^{-2}}}
$$

Thus, $\widetilde{C_{\varphi}}$ is paranormal if and only if

$$
b:=\sqrt[4]{h^{2}\left(h \circ \varphi^{-1}\right)} E(\sqrt[4]{h}) \circ \varphi^{-1}
$$

$$
D(\lambda):=a-2 b k+k^{2} \geq 0, \quad k \in(0, \infty) .
$$

Since

$$
\min _{k \in(0, \infty)} D(k)=D(b)
$$

it follows that

$$
\begin{aligned}
D(b) \geq 0 & \Longleftrightarrow a \geq b^{2} \\
& \Longleftrightarrow \frac{\sqrt{h} \circ \varphi^{-1} E\left\{h E(\sqrt{h}) \circ \varphi^{-1}\right\} \circ \varphi^{-2}}{\sqrt{E(\sqrt{h}) \circ \varphi^{-2}} \geq h \sqrt{h} \circ \varphi^{-1}\left(E(\sqrt[4]{h}) \circ \varphi^{-1}\right)^{2}} \\
& \Longleftrightarrow E\left\{h E(\sqrt{h}) \circ \varphi^{-1}\right\} \circ \varphi^{-1} \geq h \circ \varphi \sqrt{E(\sqrt{h}) \circ \varphi^{-1}}\{E(\sqrt[4]{h})\}^{2} \\
& \Longleftrightarrow E\left\{h E(\sqrt{h}) \circ \varphi^{-1}\right\} \geq\left(h \circ \varphi^{2}\right) \sqrt{E(\sqrt{h})}\{E(\sqrt[4]{h}) \circ \varphi\}^{2}, \quad \text { on } \sigma(h) .
\end{aligned}
$$

(ii) The proof is similar to the proof of part (i). Let $f \in L^{2}(\Sigma)$, then by

Lemma 2.5(ii), $\widetilde{C_{\varphi}}$ is paranormal if and only if for each $k \in(0, \infty)$,

$$
G(k):=a-2 b k+k^{2} \geq 0,
$$


where

$$
\begin{aligned}
& a:=\frac{\sqrt{h} \circ \varphi^{-1} E\left\{h E(\sqrt{h}) \circ \varphi^{-1}\right\} \circ \varphi^{-2}}{\sqrt{E(\sqrt{h}) \circ \varphi^{-2}}}, \\
& b:=\sqrt{h} \circ \varphi^{-1} E(\sqrt{h}) \circ \varphi^{-2} .
\end{aligned}
$$

Since this function takes its minimum value at $k=b$, then we have

$$
\begin{aligned}
G(b) \geq 0 & \Longleftrightarrow a \geq b^{2} \\
& \Longleftrightarrow \frac{\sqrt{h} \circ \varphi^{-1} E\left\{h E(\sqrt{h}) \circ \varphi^{-1}\right\} \circ \varphi^{-2}}{\sqrt{E(\sqrt{h}) \circ \varphi^{-2}}} \geq\left(\sqrt{h} \circ \varphi^{-1} E(\sqrt{h}) \circ \varphi^{-2}\right)^{2} \\
& \Longleftrightarrow E\left\{h E(\sqrt{h}) \circ \varphi^{-1}\right\} \circ \varphi^{-1} \geq \sqrt{h}\left(E(\sqrt{h}) \circ \varphi^{-1}\right)^{\frac{5}{2}} \\
& \Longleftrightarrow E\left\{h E(\sqrt{h}) \circ \varphi^{-1}\right\} \geq \sqrt{h \circ \varphi}\{E(\sqrt{h})\}^{\frac{5}{2}}, \quad \text { on } \sigma(h) .
\end{aligned}
$$

Thus the theorem is proved.

Recently in [23], Yamazaki introduce the notion of the *-Aluthge transformation $\widetilde{T}^{(*)}$ of $T$ by setting $\widetilde{T}^{(*)}={\widetilde{T^{*}}}^{*}=\left|T^{*}\right|^{\frac{1}{2}} U\left|T^{*}\right|^{\frac{1}{2}}$. In the following, we will obtain the $*$-Aluthge transformation of $C_{\varphi}$.

Proposition 2.7. Let $C_{\varphi}$ be a composition operator on $L^{2}(\Sigma)$. Then ${\widetilde{C_{\varphi}}}^{(*)} f=\sqrt[4]{h \circ \varphi} E\left\{\sqrt[4]{h^{3}} E(f) \circ \varphi^{-1}\right\}$

Proof. By direct computation, we get that

$$
\begin{aligned}
U\left|C_{\varphi}^{*}\right|^{\frac{1}{2}}(f)=\sqrt{h} E(\sqrt[4]{h \circ \varphi} E(f)) \circ \varphi^{-1} & =\sqrt{h} \sqrt[4]{h} E(f) \circ \varphi^{-1} \\
& =\sqrt[4]{h^{3}} E(f) \circ \varphi^{-1}
\end{aligned}
$$

Thus,

$$
{\widetilde{C_{\varphi}}}^{(*)} f=\left|C_{\varphi}^{*}\right|^{\frac{1}{2}} U\left|C_{\varphi}^{*}\right|^{\frac{1}{2}}(f)=\sqrt[4]{h \circ \varphi} E\left\{\sqrt[4]{h^{3}} E(f) \circ \varphi^{-1}\right\}
$$

\section{Examples}

In this section, we will discuss two examples.

Example 3.1. Let $X=[0,1]$ equipped with the Lebesgue measure $\mu$ on the Borel sets. Define $\varphi: X \rightarrow X$ by

$$
\varphi(x)= \begin{cases}2 x & 0 \leq x \leq \frac{1}{2} \\ 2-2 x & \frac{1}{2} \leq x \leq 1\end{cases}
$$


Then

$$
\begin{aligned}
& E(f)(x)= \frac{f(x)+f(1-x)}{2} \\
& \varphi^{2}(x)= \begin{cases}4 x & 0 \leq x \leq \frac{1}{4} \\
2-4 x & \frac{1}{4} \leq x \leq \frac{1}{2} \\
-2+4 x & \frac{1}{2} \leq x \leq \frac{3}{4} \\
4-4 x & \frac{3}{4} \leq x \leq 1 .\end{cases}
\end{aligned}
$$

A computation show that $h(x)=1$ and for each $f \in L^{2}(\Sigma)$

$$
\begin{aligned}
\left(E(f) \circ \varphi^{-1}\right)(x) & =\frac{f\left(\frac{x}{2}\right)+f\left(1-\frac{x}{2}\right)}{2}, \\
\widetilde{C_{\varphi}} f(x) & = \begin{cases}f(2 x) & 0 \leq x \leq \frac{1}{2}, \\
f(2-2 x) & \frac{1}{2} \leq x \leq 1 .\end{cases} \\
{\widetilde{C_{\varphi}}}^{*} f(x) & =\frac{f\left(\frac{x}{2}\right)+f\left(1-\frac{x}{2}\right)}{2}, \\
\left|\widetilde{C_{\varphi}}\right| f(x) & =f(x), \\
\left|{\widetilde{C_{\varphi}}}^{*}\right| f(x) & =E(f) .
\end{aligned}
$$

Also by Theorems 2.3, 2.4 and 2.6, $\widetilde{C_{\varphi}}$ is hyponormal, quasihyponormal, paranormal and also $*$-paranormal.

Example 3.2. Let $X=[1, \infty)$ be the interval equipped with the Lebesgue measure $d \mu$ on the Lebesgue measurable subsets of $X$ and let the $\varphi: X \rightarrow X$ be a non-singular measurable transformations defined by $\varphi(x)=\sqrt{x}$. Then $h(x)=2 x, E=I, h \circ \varphi(x)=2 \sqrt{x}$. In this case by Propositions 2.1, 2.2 and 2.7 , we obtain

$$
\begin{aligned}
\widetilde{C_{\varphi}} f(x) & =\sqrt[8]{x} f(\sqrt{x}) \\
{\widetilde{C_{\varphi}}}^{*} f(x) & =2 x \sqrt[4]{x} f\left(x^{2}\right), \\
{\widetilde{C_{\varphi}}}^{(*)} f(x) & =2 \sqrt[4]{x^{3} \sqrt{x}} f\left(x^{2}\right) .
\end{aligned}
$$

Also by Theorems 2.3, 2.4 and 2.6, $\widetilde{C_{\varphi}}$ is hyponormal but it is neither quasihyponormal nor $*$-paranormal. However if we change underlying space to $X=[4, \infty)$, then $\widetilde{C_{\varphi}}$ is quasihyponormal and $*$-paranormal and also hyponormal. Clearly by Theorem $2.4, \widetilde{C_{\varphi}}$ is not paranormal.

\section{REFERENCES}

[1] A. Aluthge, On $p$-hyponormal operators for $0<p<1$, Integral Equations Operator Theory, 13 (1990), 307-315.

[2] C. Burnap and I. Jung, Composition operators with weak hyponormality, J. Math. Anal. Appl, 337 (2008), no. 1, 686-694.

[3] J. T. Campbell and J. Jamison, On some classes of weighted composition operators, Glasgow Math. J, 32 (1990), 87-94. 
[4] J. T. Campbell and W. E. Hornor, Localising and seminormal composition operators on $L^{2}$, Proc. Roy. Soc. Edinburgh Sect. A ,124 (1994), 301-316.

[5] M. Cho and T. Yamazaki, Characterizations of $p$-hyponormal and weak hyponormal weighted composition operators. Acta Sci. Math. (Szeged), 76 (2010), 173-181.

[6] A. Daniluk, and J. Stochel, Seminormal composition operators induced by affine transformations, Hokkaido Math. J, 26 (1997), 377-404.

[7] H. Emamalipour, M. R. Jabbarzadeh and M. Sohrabi Chegeni, Some weak phyponormal classes of weighted composition operators. Filomat, 31.9 (2017), 26432656.

[8] T. Furuta, Invitation to linear operators, Taylor \& Francis, Ltd. London, 2001.

[9] D. Harrington and R. whitley, Seminormal composition operators, J. Operator theory, 11 (1984), 125-135.

[10] J. Herron, Weighted conditional expectation operators, Oper. Matrices, 5 (2011), 107-118.

[11] T. Hoover, A. Lambert and J. Queen, The Markov process determined by a weighted composition operator, Stdia Math. (poland), LXXII (1982), 225-235.

[12] M. R. Jabbarzadeh and M. R. Azimi, Some weak hyponormal classes of weighted composition operators, Bull. Korean. Math. Soc, 47 (2010), 793-803.

[13] D. Jung, M. Y. Lee and S. H. Lee, On classes of operators related to paranormal operators, Sci. Math. Jpn, 53 (2001), 33-43.

[14] I. B. Jung, E. Ko and C. Pearcy, Aluthge transforms of operators, Integral Equations Operator Theory, 37 (2000), 437-448.

[15] F. Kimura, Analysis of non-normal operators via Aluthge transformation, Integral Equations Operator Theory, 50 (2004), 375-384.

[16] A. Lambert, Localising sets for sigma-algebras and related point transformations, Proc. Roy. Soc. Edinburgh Sect. A, 118 (1991), 111-118.

[17] S. Panayappan and A. Radharamani, A note on $p$-*-paranormal operators and absolute $k$-*-paranormal operators. Int. J. Math. Anal, 2 (2008), 25-28.

[18] M. M. Rao, Conditional measure and applications, Marcel Dekker, New York, 1993.

[19] D. Senthilkumar and P. Maheswari Naik, Weyl's theorem for algebraically absolute( $p, r)$-paranormal operators, Banach J. Math. Anal, 5 (2011), 29-37.

[20] R. K. Singh and J. S. Manhas, Composition operators on function spaces, North Holland Math. Studies 179, Amsterdam 1993.

[21] M. Sohrabi, On the cauchy dual and complex symmetric of composition operators, International Journal of Nonlinear Analysis and Applications, 12(2021), 84-96.

[22] R. Whitley, Normal and quasinormal composition operators, Proc. Amer. Math. Soc, 70 (1978), 114-118.

[23] T. Yamazaki, Parallelisms between Aluthge transformation and powers of operators, Acta Sci. Math. (Szeged), 67(2001), 809-820.

[24] T. Yamazaki and M. Yanagida, A further generalization of paranormal operators. Sci. Math, 3(1) (2000), 23-31.

[25] T. Yamazaki, On the polar decomposition of the Aluthge transformation and related results, J. Operator Theory, 51 (2004), 303-319.

(Morteza Sohrabi) Department of Mathematics, Lorestan University, KhorRAMABAD, IRAN

Email address: sohrabi.mo@lu.ac.ir 